\title{
Population Snapshot of the Extended-spectrum $\beta$-lactamase- producing Escherichia Coli Invasive Strains Isolated From a Hungarian Hospital
}

Kinga Tóth ( $\nabla$ kingatoth91@gmail.com )

Semmelweis University: Semmelweis Egyetem https://orcid.org/0000-0002-6352-777X

Ákos Tóth

National Public Health Center

\section{Katalin Kamotsay}

Central Hospital of Southern Pest National Institute of Hematology and Infectious Disease

Viktória Németh

Central Hospital of Southern Pest National Institute of Hematology and Infectious Disease

\section{Dóra Szabó}

Semmelweis University: Semmelweis Egyetem

\section{Research}

Keywords: Escherichia coli, ST131, C1-M27, extended-spectrum $\beta$-lactamase, blaCTX-M-27, blaCTX-M-15

Posted Date: May 27th, 2021

DOI: https://doi.org/10.21203/rs.3.rs-535213/v1

License: (ㄷ) (i) This work is licensed under a Creative Commons Attribution 4.0 International License. Read Full License

Version of Record: A version of this preprint was published at Annals of Clinical Microbiology and Antimicrobials on February 10th, 2022. See the published version at https://doi.org/10.1186/s12941-022-00493-8. 


\section{Abstract}

Background:

This study was carried out to determine the prevalence and the genetic background of extended-spectrum $\beta$-lactamaseproducing Escherichia coli invasive isolates obtained from a tertiary-care hospital in Budapest, Hungary.

Methods

Between October-November 2018, all invasive ESBL-producing E. coli isolates were collected from Central Hospital of Southern Pest. The antimicrobial susceptibility testing was performed according to the EUCAST guidelines. The possible clonal relationships were investigated by core genome (cg)MLST (SeqSphere+) using whole-genome sequencing (WGS) data of isolates obtained from Illumina 251-bp paired-end sequencing. From WGS data acquired antimicrobial resistance genes and replicon types were retrieved using ResFinder3.1, PlasmidFinder2.1, and pMLST-2.0 online tools.

Results

Overall, 25 E. coli isolates were detected and six proved to be resistant to third-generation cephalosporins. Full genome sequence analysis showed that five $E$. coli isolates belonged to the ST131 clone: two to C1-M27 subclade with b/a $a_{\text {CTX-M-27 }}$ and three to $\mathrm{C} 2 / \mathrm{H} 30 \mathrm{Rx}$ subclade with b/a $a_{\mathrm{CTX}-\mathrm{M}-15}$. One isolate belonged to ST1193 with bla $\mathrm{CTX}_{\mathrm{CT}-\mathrm{M}-27}$. According to cgMLST, all $\mathrm{C} 2 / \mathrm{H} 30 \mathrm{Rx}$ isolates formed a cluster ( $\leq 6$ allele differences), while the bla $a_{\mathrm{CTX}-\mathrm{M}-27}$-producing C1-M27 isolates differed at least 35 alleles from each other. Both C2/H30Rx and C1-M27 ST131 isolates harbored similar antimicrobial resistance gene sets. However, only $\mathrm{C} 2 / \mathrm{H} 30 \mathrm{Rx}$ isolates had the $\mathrm{qnrB}$ and $\mathrm{aac}(3)$-lla. All isolates showed resistance against ceftriaxone, cefotaxime, and ciprofloxacin, and the $\mathrm{C} 2 / \mathrm{H} 30 \mathrm{Rx}$ isolates were also resistant to gentamicin, tobramycin, and ceftazidime.

Conclusions

Out of six third-generation cephalosporins-resistant, invasive E. coli, five belonged to the S131clone. This study indicates, that the C2/H30Rx and C1-M27 subclades of the ST131 appear to be the dominant clones collected in a Hungarian hospital.

\section{Background}

Recently the burden of disease caused by third-generation cephalosporin-resistant Escherichia coli increased the most, in terms of the number of infections and the number of deaths in Europe [1]. Its global spread is associated with the sequence type 131 (ST131) high-risk clonal group. Members of ST131 are primarily extraintestinal pathogenic E. coli (ExPEC) harbor various virulence genes that allow them to cause severe extraintestinal infections, include bloodstream infections, urinary tract infections, pneumonia, and neonatal meningitis [2].

E. coli ST131 high-risk clones connected with extended-spectrum $\beta$-lactamase (ESBL) production and fluoroquinolone resistance causing multidrug-resistant infections belong to the most prevalent clade $\mathrm{C}$ associated with fimH30. Within $\mathrm{C} / \mathrm{H} 30$, two subclades were emerging in the 2000s: C1/H30R subclade with fluoroquinolone resistance (FQ-R) and C2/H30Rx subclade with bla $a_{\mathrm{CTX}-\mathrm{M}-15}$, and FQ-R [3, 4]. In the late 2000s, C1-M27 clade with bla $\mathrm{CTX}_{\mathrm{M}-27}$ of C1/H30R became dominant in Asia and showed global distribution like the $\mathrm{C} 2 / \mathrm{H} 30 \mathrm{Rx}$ did before [4]. The expansion of the $\mathrm{C} 1$ and $\mathrm{C} 2$ subclades was driven by the acquisition of CTX-M genes containing IncF plasmids which evolve with the clone. The $\mathrm{F} 2: \mathrm{A} 1$ :B- plasmid carrying the bla $\mathrm{C}_{\mathrm{CTX}-\mathrm{M}-15}$ gene is associated with the $\mathrm{C} 2 / \mathrm{H} 30 \mathrm{Rx}$ subclade and the $b / a_{\mathrm{CTX}-\mathrm{M}-27}$ encoding $\mathrm{F} 1: A 2: B 20$ plasmid is associated with the C1-M27 subclade $[5,6]$. The other recently emerging high-risk clones among ESBLproducing E. coli are ST10, ST38, ST69, ST155, ST315, ST405, ST410, ST648, and ST1193 [7-10].

Previous studies have shown that the proportion of third-generation cephalosporin-resistance among invasive $E$. coli has risen in Hungary from $5.1 \%$ in 2006 to $22.6 \%$ in 2018 [11, 12]. However, there are few data available on the incidence of 
third-generation cephalosporin-resistant invasive E. coli from Hungary, and its genomic epidemiology and no prospective study was made before.

This study was designed to determine the incidence of third-generation cephalosporin-resistant invasive $E$. coli collected from a Hungarian hospital; and to perform genomic typing of identified ESBL-producing isolates in order to characterize their genetic background.

\section{Methods}

Study design

Central Hospital of Southern Pest National Institute of Hematology and Infectious Diseases is a tertiary-care hospital in Budapest, Hungary, with a capacity of approximately 1400 beds. Between October-November 2018, all invasive consecutive non-duplicate clinical isolates of ESBL-producing E. coli were collected in the Central Hospital of Southern Pest.

Bacterial isolates and antimicrobial susceptibility testing

All isolates were identified using matrix-assisted laser desorption ionization time-of-flight mass spectrometry (MALDI-TOF). The antibiotic susceptibility test of putative ESBL-producing isolates was performed to ceftriaxone, ceftazidime, cefotaxime, gentamicin, amikacin, tobramycin, fosfomycin, ceftazidime/avibactam, tigecycline, ertapenem by gradient MIC tests (Liofilchem, Roseto degli Abruzzi, Italy); and to imipenem, meropenem, ciprofloxacin, colistin - by broth microdilution and interpreted using EUCAST guidelines [13].

Molecular characterization

Genomic DNA analysis of the isolates was performed by whole-genome sequencing (WGS) using Illumina MiSeq 251-bp paired-end sequencing. Raw data were processed by Ridom SeqSphere + and assembly was performed using Velvet 1.1.04 [14]. The possible clonal relationships were investigated by core genome (cg)MLST (SeqSphere+). Cluster type (CT) for $E$. coli was defined as isolates attributed to the same clone with $\leq 10$ differing alleles (CT threshold) within a group. The ST131 isolates were compared to three international ST131 E. coli genomes (C1-M27: EC81009 [15] and H105 [16], C2/H30Rx: JJ2434 [17]) obtained from GenBank, to reveal the relatedness to the subclades of the ST131 clone. The replicon types were retrieved using PlasmidFinder2.1 and pMLST-2.0 [18] online tools at the Center for Genomic Epidemiology (CGE, http://genomicepidemiology.org/). The isolates were compared to the pEC-81009 (Group 1 replicon: IncFII_1, IncFIA_2, IncFIB_20), to the uk_P46212 plasmid (Group 2 of replicon: IncFII_2, IncFIA_1) and pU1 (F1:A1:B16) reference plasmid sequences to determine the coverage and identity between them [6]. The reads were trimmed (the quality threshold of 30 reads was filtered for a window of 20 bases based on filtering) and mapped to the reference plasmid sequences by BWA (SeqSphere+) and the mapped sequences were analyzed by BLASTn. The pEC-81009 (C1 clades reference plasmid, Group 1 of replicon: IncF[F1:A2:B20]; CP021180), the uk_P46212 plasmid (C2 clades reference plasmid, Group 2 of replicon: IncF[F2:A1:B-]; CP013657), and the pU1 (MK295825) reference plasmid sequences, and their terminologies and definitions were obtained from a meta-analysis of Kondratyeva et al. [6], and the GenBank. The presence of the M27PP1 prophage-like region characteristic was investigated by comparing it with the M27PP1 prophage-like region sequences of KUN5781 [19]. From WGS data the acquired antimicrobial resistance genes were identified using ResFinder3.1 [20] CGE online tool. The raw reads are available on the Sequence Read Archive (SRA) database under the BioProject number PRJNA683640.

\section{Results}

Clinical epidemiology

Twenty-five $E$. coli were isolated from haemoculture in the study period. The overall incidence density was 0.48 per 1,000 patient days and the incidence was 3.31 per 1,000 hospital admission. Six from 25 isolates (24\%) proved to be resistant to 
third-generation cephalosporins. Phenotypic detection tests showed that all of them were ESBL producers.

Antimicrobial susceptibility

The isolates were resistant to ceftriaxone, cefotaxime, and ciprofloxacin but remained susceptible to colistin, fosfomycin, ceftazidime-avibactam, and carbapenems (Table 1). The Ec1, Ec2, and Ec3 showed resistance to ceftazidime, gentamicin, and tobramycin too. Moreover, the Ec3, Ec5 isolates showed resistance to tigecycline and Ec6 to amikacin.

Table 1

Antimicrobial susceptibility of the ESBL-producing E. coli isolates (MIC (mg/L)

\begin{tabular}{|lllllllllllllll|}
\hline Isolates & CIP & CRO & CAZ & CTX & CZA & GM & AK & TM & TGC & FOS & COL & ETP & MEM & IMI \\
\hline Ec1 & 256 & 256 & 32 & $\underset{2}{2}$ & 1 & 16 & 2 & 8 & 0.25 & 0,25 & 0.125 & 0.125 & 0.064 & 0.125 \\
\hline Ec2 & 64 & 256 & 32 & $\begin{array}{l}\geq \\
256\end{array}$ & 0,5 & 64 & 4 & 32 & 0.5 & 1 & 0.25 & 0.125 & 0.125 & 0.125 \\
\hline Ec3 & 64 & 256 & 32 & $\geq$ & 1 & 64 & 2 & 8 & 1 & 4 & 0.125 & 0.125 & 0.064 & 0.125 \\
\hline Ec4 & 64 & 256 & 4 & 64 & 0.5 & 2 & 4 & 1 & 0.25 & 2 & 0.25 & 0.008 & 0.032 & 0.125 \\
\hline Ec5 & 64 & 256 & 4 & 64 & 0.25 & 1 & 4 & 2 & 1 & 4 & 0.25 & 0.008 & 0.032 & 0.125 \\
\hline Ec6 & 16 & 256 & 4 & $\geq$ & 0.125 & 0.5 & 16 & 1 & 0.125 & 1 & 0.064 & 0.016 & 0.032 & 0.125 \\
\hline
\end{tabular}

The used antibiotics: ciprofloxacin (CIP), ceftriaxone (CRO), ceftazidime (CAZ), cefotaxime (CTX), ceftazidime/avibactam (CZA), gentamicin (GM), amikacin (AK), tobramycin (TM), tigecycline (TGC), fosfomycin (FOS), colistin (COL), ertapenem (ETP), meropenem (MEM), imipenem (IMI). The Ec1, Ec3, Ec3 correspond to ST131 C2/H30Rx isolates, Ec4, Ec5 correspond to ST131 C1-M27 isolates and Ec6, correspond to ST1193.

Molecular characterization

The six ESBL-producing E. coli isolates could be assigned to two different sequence types (ST) by MLST. Of the six isolates, five (Ec1-Ec5) belonged to the globally dominant ST131 clone (025:H4 serotype), which comprised two distinguished groups of strains with different characteristics. The first group contains two isolates with $b / a_{\mathrm{CTX}-\mathrm{M}-15}$ and one isolate with $b / a_{\mathrm{CTX}-\mathrm{M}-15}$ and $b / a_{\mathrm{TEM}-1 \mathrm{~B}}$ while the two other isolates harbored $b / a_{\mathrm{CTX}-\mathrm{M}-27} \mathrm{ESBL}$-genes. The remained one isolate belonged to the ST1193 with bla $a_{\mathrm{CTX}-\mathrm{M}-27}$. Analysis of genetic relatedness between the strains was performed by cgMLST. The five ST131 isolates were compared to one C2/H30Rx (JJ2434) and two C1-M27 (H105, EC81009) international ST131 ESBL-producing E. coli isolates, to determine the subclade-specific relatedness of the isolates within the ST131 (Fig. 1). The international isolate JJ2434 and the three CTX-M-15-producing isolates (named the first group below) belonged ST131 C2/H30Rx subclade. The H105 and EC81009 isolates with the two CTX-M-27-producing isolates from this study belonged to the ST131 C1-M27 subclade. The Ec4 and Ec5 harboured exclusively the C1-M27 clade-specific M27PP1 prophage-like genomic island.

According to cgMLST (Fig. 2), all the three Hungarian ST131 E. coli isolates producing bla $a_{\mathrm{CTX}-\mathrm{M}-15}$ formed a close cluster ( $\leq 6$ allele differences), while the C1-M27 isolates producing b/a ${ }_{\mathrm{CTX}-\mathrm{M}-27}$ differed at 35 alleles from each other. The ST1193 isolates showed $\geq 2209$ allele differences from any others.

The isolates of each group belonged to ST131 harboured very similar resistance gene sets. The characteristics of resistome and plasmid replicon types of the isolates are shown in Table 2. Although, there are some differences between the two ST131 subclades in terms of resistance genes like the $q n r B$ (FQ R) and aac(3)-Ila (aminoglycoside R) which were present 
only in C2/H30Rx isolates. The ST1193 isolate harboured L416F mutation in parE, and also had no qnrB and aac(3)-Ila genes. Three plasmid families were detected: IncF, IncQ, and Col-like. The IncF replicons were present in every isolate, but only the C1-M27 ST131 isolates (EC4, EC5) carried the Group1 replicons (IncFII_1, IncFIA_2, IncFIB_20). The coverage of the Group 1 (F1:A1:B20) reference plasmid by the reads of Ec4 and Ec5 was 96\%, and 72\%, respectively (Table 3 ). None of the C2/H30Rx isolates (EC1-EC3) co-harboured the two reference replicons of Group2 (IncFII_2, IncFIA_1). The Ec2 did not carry any IncFIA replicon but carried the IncFII_2 replicon. The coverage of the Group2 (F2:A1:B-) reference plasmid by the reads of Ec1, Ec2, and Ec3 was 70\%, 47\%, and 70\%, respectively. The reads of Ec1 and Ec3 covered pU1 plasmid to a higher breadth compared to Group2 reference ( $\geq 91 \%$ vs $70 \%$ ). The ST1193 isolates harbored IncFII_4-like, IncF1A_1, IncFIB_10, and the repCol156, and repCol(BS512) replicons. The Col(pHAD28), Col156, and Col8282 replicons were at least present in one of the ST131 isolates. 
Table 2

Resistome and plasmid replicons of the ESBL-producing ExPEC strains

\begin{tabular}{|c|c|c|c|c|c|c|c|}
\hline \multicolumn{2}{|l|}{ Isolate } & \multirow{2}{*}{$\begin{array}{l}\text { Ec1 } \\
+\end{array}$} & \multirow{2}{*}{$\begin{array}{l}\text { Ec2 } \\
+\end{array}$} & \multirow{2}{*}{$\begin{array}{l}\text { Ec3 } \\
+\end{array}$} & \multirow[t]{2}{*}{ Ec4 } & \multirow[t]{2}{*}{ Ec5 } & \multirow[t]{2}{*}{ Ec6 } \\
\hline$\beta$-lactam & $\begin{array}{l}\text { blaCTX-M- } \\
15\end{array}$ & & & & & & \\
\hline & $\begin{array}{l}\text { blacTX-M- } \\
27\end{array}$ & & & & + & + & + \\
\hline & $\begin{array}{l}\text { blaTEM- } \\
1 \mathrm{~B}\end{array}$ & & + & & & & \\
\hline \multirow[t]{7}{*}{ Fluoroquinolone } & $\begin{array}{l}\text { gyrA* } \\
\text { (S83L) }\end{array}$ & + & + & + & + & + & + \\
\hline & $\begin{array}{l}\text { gyrA* } \\
\text { (D87N) }\end{array}$ & + & + & + & + & + & + \\
\hline & $\begin{array}{l}\text { parC* } \\
\text { (S80I) }\end{array}$ & + & + & + & + & + & + \\
\hline & $\begin{array}{l}\text { parC* } \\
\text { (E84V) }\end{array}$ & + & + & + & + & + & \\
\hline & $\begin{array}{l}\text { parE* } \\
(\text { (I529L) }\end{array}$ & + & + & + & + & + & \\
\hline & $\begin{array}{l}\text { parE* } \\
\text { (L416F) }\end{array}$ & & & & & & + \\
\hline & qnrB19 & + & + & + & & & \\
\hline \multirow[t]{2}{*}{ MLS } & $m p h(A)$ & + & & + & + & + & + \\
\hline & $m d f(A)$ & + & + & + & + & + & + \\
\hline \multirow[t]{2}{*}{ Sulphonamide } & sul1 & + & + & + & + & + & + \\
\hline & sul2 & + & + & + & + & + & + \\
\hline Tetracycline & $\operatorname{tet}(A)$ & + & + & + & + & + & + \\
\hline \multirow[t]{2}{*}{ Trimethoprim } & dfrA17 & + & + & + & + & + & + \\
\hline & $d f r A 1$ & & + & & & & \\
\hline \multirow[t]{5}{*}{ Aminoglycoside } & $\operatorname{ant}\left(3^{\prime \prime}\right)-l a$ & & + & + & & & \\
\hline & aadA5 & + & & + & + & + & + \\
\hline & $a p h\left(3^{\prime \prime)}-1 b\right.$ & + & + & + & + & + & + \\
\hline & $\operatorname{aph}(6)-I d$ & + & + & + & + & + & + \\
\hline & $\operatorname{aac}(3)-1 / a$ & + & + & + & & & \\
\hline \multirow{6}{*}{\multicolumn{2}{|c|}{ Plasmid replicon type }} & IncFIB & IncFIB & IncFIB & IncFIB & IncFIB & IncFIB \\
\hline & & IncFIA & IncFII & IncFIA & IncFIA & IncFIA & IncFIA \\
\hline & & IncFII & IncQ1 & IncFII & IncFII & IncFII & IncFII \\
\hline & & Col156 & Col8282 & Col156 & Col156 & & Col156 \\
\hline & & Col(pHAD28) & Col(pHAD28) & Col(pHAD28) & Col8282 & & Col(BS512) \\
\hline & & & & & Col(pHAD28 & & \\
\hline
\end{tabular}


Acquired antibiotic resistance genes and mutations of the isolates. The Ec1, Ec2, Ec3 correspond to ST131 C2/H30Rx isolates, Ec4, Ec5 correspond to ST131 C1-M27 isolates and Ec6, correspond to ST1193. The + indicates the presence of a gene, * the mutations in quinolone resistance determining region, and $\ddagger$ the plasmid-mediated quinolone resistance.

Table 3

The coverage and identity of the ST131 isolates and the references plasmids

\begin{tabular}{|lllll|}
\hline Isolates & ST131 subclade & Reference plasmid sequence & Coverage & Identity \\
\hline Ec1 & C2/H30Rx & Group2 & $70 \%$ & $97.89 \%$ \\
\cline { 3 - 5 } & & $\mathrm{pU1}$ & $91 \%$ & $99.29 \%$ \\
\hline Ec2 & C2/H30Rx & Group2 & $47 \%$ & $99.20 \%$ \\
\cline { 3 - 5 } & & $\mathrm{pU1}$ & $52 \%$ & $96.67 \%$ \\
\hline Ec3 & C2/H30Rx & Group2 & $70 \%$ & $97.21 \%$ \\
\cline { 3 - 5 } & & pU1 & $92 \%$ & $99.87 \%$ \\
\hline Ec4 & C1-M27 & Group1 & $96 \%$ & $99.93 \%$ \\
\hline
\end{tabular}

Group1 plasmid refers as CTX-M-27-encoding IncF[F1:A2:B20] plasmids, Group2 plasmid as CTX-M-15-encoding IncF[F2:A1:B-] plasmids, and pU1 as IncF[F1:A1:B16] plasmid. The Ec1, Ec3, Ec3 correspond to ST131 C2/H30Rx isolates, Ec4, Ec5 correspond to ST131 C1-M27 isolates.

\section{Discussion And Conclusion}

In our study, the incidence of invasive E. coli cases was 3.31 per 1,000 hospital admission and the incidence density was 0.48 per 1,000 patient-days. There are a few previous data regarding the incidence of invasive $E$. coli infections in Hungary. In a study, Trethon et al. investigated the bloodstream infection of cardiothoracic surgery patients at the Gottsegen György Hungarian Institute of Cardiology between 1999 and 2000. The E. coli bloodstream infection incidence was 1.75 per 1000 admissions [21]. A similar increasing incidence was observed in southeast Sweden where Holmbom et al. investigated the $E$. coli bloodstream infection from four hospitals between 2008 and 2016, the incidence of $E$. coli bacteremia varying between 1.92-3.9 per 1000 hospital admissions [22].

Martelius et al. studied the invasive E. coli isolates collected by 17 Finnish acute care hospitals during 1999-2013, where the overall incidence density was 0.1 per 1,000 patient days and the incidence density of nosocomial BSIs caused by thirdgeneration cephalosporin-resistant $E$. coli cases was 0.008 per 1,000 patient-days [23]. Moreover, from a Japan study, the BSI E. coli incidence per 1,000 patient-days was increased from 0.13 to 0.17 between 2008-2012 [24]. In our study, the incidence rate was higher than in the previous studies above.

Six from the $25 \mathrm{E}$. coli isolates (24\%) proved to be 3rd generation cephalosporin-resistant and ESBL-producers. In 2010, Pál T. et al. found 26 ESBL-producers among $117 \mathrm{E}$. coli isolates (22.2\%) from bloodstream infections collected in three university hospitals in Hungary between March and November 2010, where nine of them belonged to ST131 clone carrying

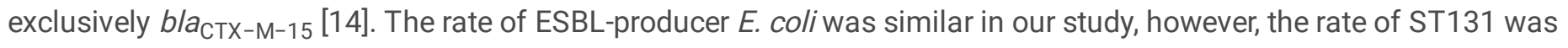
higher (83.3\%vs.34.6\% ). Furthermore, in our study two of the five isolates harbouring b/a $\mathrm{CTX}_{\mathrm{CT}-\mathrm{M}-27}$ gene belonged to the C1M27 subclade. The first ESBL-producing invasive E. coli isolates belonged to the C1-M27 subclade were detected in 2015 in Hungary (unpublished data, the National Public Health Center). In 2018, according to an observational study of Jánvári et al., 44.6\% (75/168) of invasive ESBL-producing E. coli isolates investigated at the National Public Health Center belonged to the ST131 clone, where the ratio of C2/H30Rx and C1-M27 was 1 to 0.8 [25]. 
In this study, ST131, including the C1-M27 (2 isolates) and the C2/H30Rx subclades (3 isolates) proved to be the dominant multidrug-resistant $E$. coli clone in invasive infections. The three ST131 C2/H30Rx isolates formed a relatively close cluster ( $\leq 6$ allele differences), and this could suggest an undetected outbreak affected two hospital wards. However, based on the results of plasmid replicon analysis it was unlikely.

All isolates were resistant to ceftriaxone and cefotaxime. The isolates with CTX-M-15 ESBL were resistant to ceftazidime, while the CTX-M-27 expressing isolates remained susceptible, probably because these enzymes hydrolyze ceftazidime poorly. Moreover, the $\mathrm{C} 2 / \mathrm{H} 30 \mathrm{Rx}$ isolates showed resistance to tobramycin and gentamicin probably because they have AAC(3)-Ila aminoglycoside-modifying enzyme[26].

We found one isolate that belonged to the emerging ST1193 global high-risk clone [27]. To the best of our knowledge, this study revealed for the first time the presence of the ST1193 E. coli in Hungary. This clone has been widely spread in Asia [28, 29] The ST1193 was the second most frequent ST (23.7\% of 99 isolates) after ST131 (37.3\%) among ciprofloxacin-resistant invasive E. coli isolates in the Tertiary Care University Hospital in Korea [30]. In Europe, it has been described in Germany where the rate of ESBL-producing E. coliST1193 was 0.6\% (3/495) among all E. coli infections [31]. In a South-West England study, 11 of 836 E. coli from urine samples belonged to ST1193 [32].

It has been known that the narrow-host-range IncF type plasmids have been evolved along with fluoroquinolone-resistant ST131 H30 clades. The F2:A1:B- plasmid is associated with the C2/H30Rx subclade, whereas the F1:A2:B20 plasmid is strongly associated with the $\mathrm{C} 1 / \mathrm{H} 30 \mathrm{R}$ subclade [5]. The presence of Col-like plasmids has been reported also in ST131, and in ST1193 E. coli clones, however, the exact role of these plasmids is not clear [6]. In our study, each of the isolates had various Inc-type $(F, Q)$ replicons and except Ec5, they had a Col-type (pHAD28, BS512, 156, 8282) replicons as well. The C1M-27 isolates carried the Group1 plasmid replicons (IncFII_1, IncFIA_2, IncFIB_20) and the reads of Ec4 isolate covered the Group1 reference plasmid to a higher breadth compared to Ec5 (96\% vs 72\%). The C2H30/Rx isolates did not co-encode together the standard Group2 plasmid replicons (IncFII_2, IncFIA_1). The reads of Ec1 and Ec3 shared 70\% coverage with F2:A1:B- Group2 plasmid and Ec2 shared 47\%. Ec1 and Ec3 had identical replicons to the pU1 plasmid (pMLST: F1:A1:B16) [6]. The coverage of pU1 by Ec1 and Ec3 reads was $91 \%$ and $92 \%$, respectively. These findings suggest that these two isolates harbored a similar plasmid like the pU1. The ST1193 isolates had different replicons (IncFII_4-like, IncFIB_10) than in Group1 and Group2 plasmids.

It is not completely understood, which key factors determine the successful dissemination of the ST131 C2/H30Rx and C1/H30R (C1-M27) clones worldwide, besides the antibiotic genes, but the virulence armament probably has a really important part in it. It has been described that ST131 clones can transmit among human and animal hosts [33]. Based on a study from Israel, the CTX-M-27-producing ST131 subclones had a higher transmission rate than CTX-M-15-producing ST131 subclones [34]. At this number of isolates, we cannot estimate if the selection effect of the antibiotic consumption influences the dissemination of the ST131 subclades, especially the third-generation cephalosporins, because it needs further investigation.

This study has several limitations, like small sample size and a two months time limit. However, it gives an insight into the incidence and genomic characteristics of ESBL-producing E. coli isolates in a Hungarian hospital. It highlights the dominance of the ST131 clones and the C1-M27 and C2/H30Rx subclone in invasive infections. Although, further national studies are needed with a larger collection of isolates belonging to the two subclades to determine the possible factors (virulence, antibiotic resistance) that can influence their dissemination.

\section{Abbreviations}

BSI

bloodstream infection

cgMLST 
core genome multilocus sequence typing

CT

cluster-type

ESBL

extended-spectrum $\beta$-lactamase

EUCAST

European Committee on Antimicrobial Susceptibility Testing

ExPEC

extraintestinal pathogenic E. coli

FQ-R

fluoroquinolone resistance

MALDI-TOF

matrix-assisted laser desorption ionization time-of-flight mass spectrometry

MIC

minimum inhibitory concentration

MLST

multilocus sequence typing

SRA

Sequence Read Archive

ST

sequence typing

WGS

whole-genome sequencing

\section{Declarations}

\section{Authors' contributions}

KT and ÁT contributed to the study design. KT performed the experiments and wrote the manuscript. KK and VN collected the samples. TK, ÁT, and DSz drafted and revised the manuscript. All authors read and approved the final manuscript.

\section{Acknowledgements}

Dr. Ivelina Damjanova provided helpful suggestions and assisted by critically reading the manuscript.

\section{Competing interests}

All authors declare that they have no competing interests.

\section{Availability of data and material}

The data set supporting the results of this article are included within the article.

\section{Consent for publication}

All authors approve the publication of this work. 


\section{Ethics approval and consent to participate}

This is a retrospective study, not directly associated with patients and it was consistent with the principles of the Helsinki Declaration.

\section{Funding}

This study was financially supported by OTKA Hungarian Scientific Fund, grant number: 108481.

\section{References}

1. Cassini A, Högberg LD, Plachouras D, Quattrocchi A, Hoxha A, Simonsen GS, et al. Attributable deaths and disabilityadjusted life-years caused by infections with antibiotic-resistant bacteria in the EU and the European Economic Area in 2015: a population-level modelling analysis. Lancet Infect Dis. 2019;19:56-66.

2. Smith JL, Fratamico PM, Gunther NW. Extraintestinal Pathogenic Escherichia coli. Foodborne Pathog Dis. 2007;4:13463.

3. Pitout JDD, DeVinney R. Escherichia coli ST131: A multidrug-resistant clone primed for global domination. F1000Research. 2017;6:1-7.

4. Mathers AJ, Peirano G, Pitout JDD. The role of epidemic resistance plasmids and international high-risk clones in the spread of multidrug-resistant Enterobacteriaceae. Clin Microbiol Rev. 2015;28:565-91.

5. Johnson TJ, Danzeisen JL, Youmans B, Case K, Llop K, Munoz-Aguayo J, et al. Separate F-Type Plasmids Have Shaped the Evolution of the $\mathrm{H} 30$ Subclone of Escherichia coli Sequence Type 131. mSphere. American Society for Microbiology; 2016;1.

6. Kondratyeva K, Salmon-Divon M, Navon-Venezia S. Meta-analysis of Pandemic Escherichia coli ST131 Plasmidome Proves Restricted Plasmid-clade Associations. Sci Rep. 2020;10:1-11.

7. Peirano G, Van Der Bij AK, Gregson DB, Pitout JDD. Molecular epidemiology over an 11-year period (2000 to 2010) of extended-spectrum $\beta$-lactamase-producing Escherichia coli causing bacteremia in a centralized Canadian region. J Clin Microbiol. 2012;50:294-9.

8. Roer L, Overballe-Petersen S, Hansen F, Schønning K, Wang M, Røder BL, et al. Escherichia coli Sequence Type 410 Is Causing New International High-Risk Clones. mSphere. 2018;3:e00337-18.

9. Baquero F, Tedim AP, Coque TM. Antibiotic resistance shaping multi-level population biology of bacteria. Front Microbiol. 2013;4:1-15.

10. Schaufler K, Semmler T, Wieler LH, Trott DJ, Pitout J, Peirano G, et al. Genomic and Functional Analysis of Emerging Virulent and Multidrug-Resistant Escherichia coli Lineage Sequence Type 648. Antimicrob Agents Chemother. 2019;63:1-12.

11. Tóth Á, Juhász-Kaszanyitzky É, Mag T, Hajbel-Vékony G, Pászti J, Damjanova I. Characterization of extended-spectrum $\beta$-lactamase (ESBL) producing Escherichia coli strains isolated from animal and human clinical samples in Hungary in 2006-2007. Acta Microbiol Immunol Hung. 2013;60:175-85.

12. European Centre for Disease Prevention and Control. Surveillance of antimicrobial resistance in Europe 2018. Stockholm: ECDC; 2019. Sep.

13. EuCAST. EuCAST breakpoints. Eur Comm Antimicrob Susceptibility Testing. 2018;0-77.

14. Zerbino DR, Birney E. Velvet: Algorithms for de novo short read assembly using de Bruijn graphs. Genome Res. 2008;18:821-9.

15. Mutti M, Sonnevend Á, Pál T, Junttila S, Ekker H, Galik B, et al. Complete genome sequence of Escherichia coli 81009, a representative of the sequence type 131 C1-M27 clade with a multidrug-resistant phenotype. Genome Announc. 
2018;6:1-2.

16. Ghosh H, Bunk B, Doijad S, Schmiedel J, Falgenhauer L, Spröer C, et al. Complete genome sequence of blaCTX-M-27encoding Escherichia coli strain H105 of sequence type 131 lineage C1/H30R. Genome Announc. 2017;5:30-1.

17. Johnson TJ, Danzeisen JL, Youmans B, Case K, Llop K, Munoz-Aguayo J, et al. Separate F-Type Plasmids Have Shaped the Evolution of the H30 Subclone of Escherichia coli Sequence Type 131. mSphere. 2016;1:1-15.

18. Sahare P, Moon A. In silico modelling of $\beta$-lactam resistant Enterococcus faecalis PBP4 and its interactions with various phyto-ligands. Int J Pharm Pharm Sci. 2016;8:151-5.

19. Matsumura Y, Pitout JDD, Gomi R, Matsuda T, Noguchi T, Yamamoto M, et al. Global Escherichia coli Sequence Type 131 Clade with bla CTX-M-27 Gene. Emerg Infect Dis. 2016;22:1900-7.

20. Bortolaia V, Kaas RS, Ruppe E, Roberts MC, Schwarz S, Cattoir V, et al. ResFinder 4.0 for predictions of phenotypes from genotypes. J Antimicrob Chemother. 2020;75:3491-500.

21. Trethon A, Prinz G, Varga A, Kocsis I. Characteristics of nosocomial bloodstream infections at a Hungarian cardiac surgery centre. Acta Microbiol Immunol Hung. 2012;59:271-83.

22. Holmbom M, Möller V, Nilsson LE, Giske CG, Rashid MU, Fredrikson M, et al. Low incidence of antibiotic-resistant bacteria in south-east Sweden: An epidemiologic study on 9268 cases of bloodstream infection. PLoS One. 2020;15:116.

23. Martelius T, Jalava J, Kärki T, Möttönen T, Ollgren J, Lyytikäinen O. Nosocomial bloodstream infections caused by Escherichia coli and Klebsiella pneumoniae resistant to third-generation cephalosporins, Finland, 1999-2013: Trends, patient characteristics and mortality. Infect Dis (Auckl). 2016;48:229-34.

24. Nagao M. A multicentre analysis of epidemiology of the nosocomial bloodstream infections in Japanese university hospitals. Clin Microbiol Infect. European Society of Clinical Infectious Diseases; 2013;19:852-8.

25. Jánvári L, Damjanova I, Topf J, Mag T, Mestyan G, Szolyka G, et al. Countrywide dissemination of CTX-M-27-producing Escherichia coli ST131 subclade. ECCMID 2018.

26. Shaw KJ, Rather PN, Hare RS, Miller GH. Molecular genetics of aminoglycoside resistance genes and familial relationships of the aminoglycoside-modifying enzymes. Microbiol Rev. 1993;57:138-63.

27. Johnson TJ, Elnekave E, Miller EA, Munoz-Aguayo J, Flores Figueroa C, Johnston B, et al. Phylogenomic Analysis of Extraintestinal Pathogenic Escherichia coli Sequence Type 1193, an Emerging Multidrug-Resistant Clonal Group. Antimicrob Agents Chemother. 2018;63:1-15.

28. Al-Farsi HM, Camporeale A, Ininbergs K, Al-Azri S, Al-Muharrmi Z, Al-Jardani A, et al. Clinical and molecular characteristics of carbapenem non-susceptible Escherichia coli: A nationwide survey from Oman. PLoS One. Public Library of Science; 2020;15.

29. Wu J, Lan F, Lu Y, He Q, Li B. Molecular characteristics of ST1193 clone among phylogenetic group B2 Non-ST131 fluoroquinolone-resistant Escherichia coli. Front Microbiol. Frontiers Media S.A.; 2017;8.

30. Kim Y, Oh T, Nam Y, Cho S, Lee H. Prevalence of ST131 and ST1193 Among Bloodstream Isolates of Escherichia coli not Susceptible to Ciprofloxacin in a Tertiary Care University Hospital in Korea, 2013 - 2014. Clin Lab. 2017;63.

31. Valenza G, Werner M, Eisenberger D, Nickel S, Lehner-Reindl V, Höller C, et al. First report of the new emerging global clone ST1193 among clinical isolates of extended-spectrum $\beta$-lactamase (ESBL)-producing Escherichia coli from Germany. J Glob Antimicrob Resist. Taibah University; 2019;17:305-8.

32. Findlay J, Gould VC, North P, Bowker KE, Williams MO, MacGowan AP, et al. Characterization of cefotaxime-resistant urinary Escherichia coli from primary care in South-West England 2017-18. J Antimicrob Chemother. 2020;75:65-71.

33. Kidsley AK, White RT, Beatson SA, Saputra S, Schembri MA, Gordon D, et al. Companion Animals Are Spillover Hosts of the Multidrug-Resistant Human Extraintestinal Escherichia coli Pandemic Clones ST131 and ST1193. Front Microbiol. 2020;11:1-10. 
34. Adler A, Gniadkowski M, Baraniak A, Izdebski R, Fiett J, Hryniewicz W, et al. Transmission dynamics of ESBL-producing Escherichia coli clones in rehabilitation wards at a tertiary care centre. Clin Microbiol Infect. 2012;18.

\section{Figures}

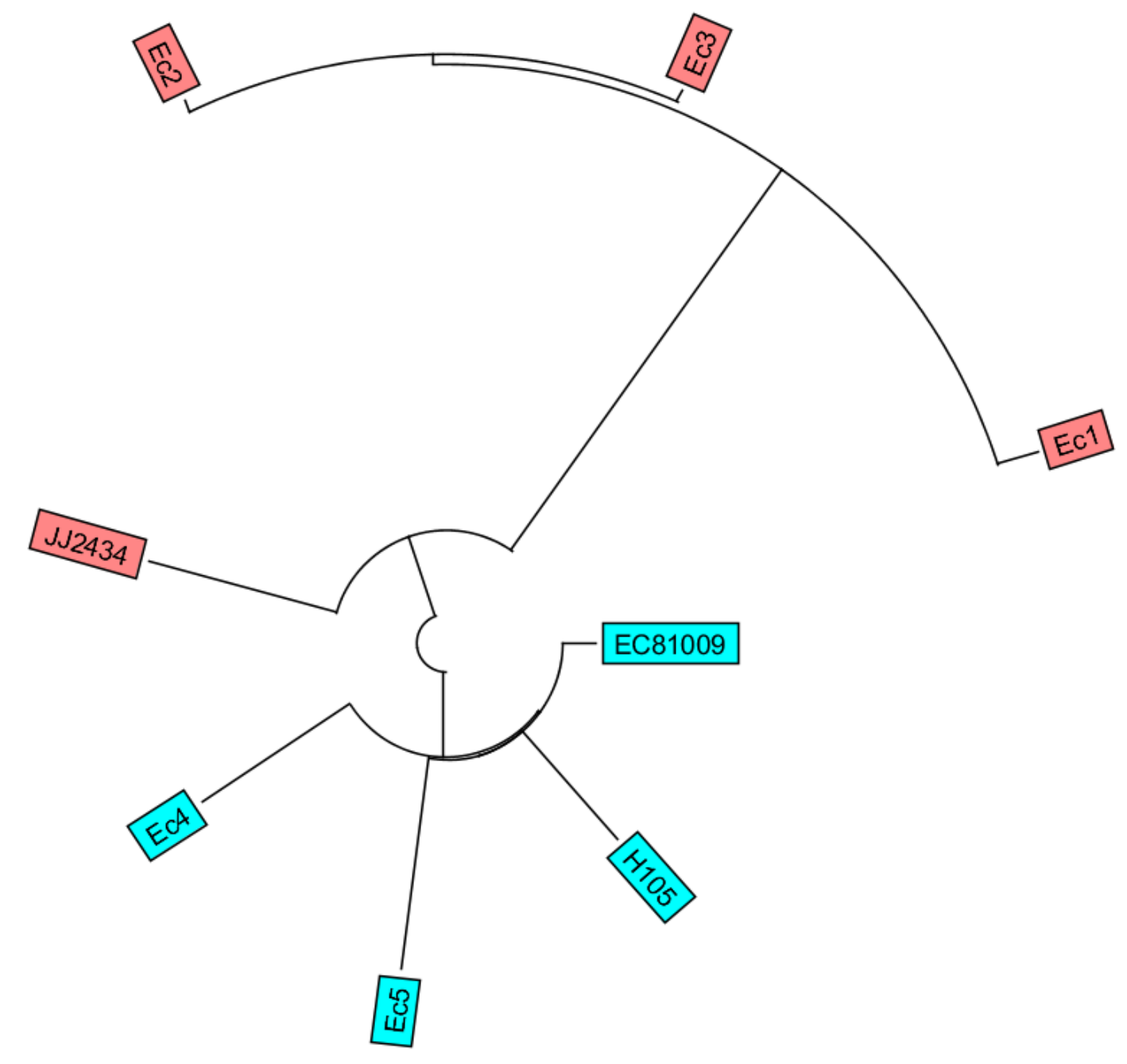

\section{Figure 1}

The ST131 subclade-specific phylogenetic relationship of the Escherichia coli isolates Figure 1 legend: The unrooted circular phylogenetic tree of ST131 Escherichia coli isolates based on the Ridom ${ }^{\text {TM }}$ SeqSphere+ core genome multilocus sequencing typing (cgMLST) including 2517 alleles. The blue colour corresponds to Sequence Type 131 C1-M27 subclade, while the red to Sequence Type $131 \mathrm{C} 2 / \mathrm{H} 30 \mathrm{Rx}$ subclade. 


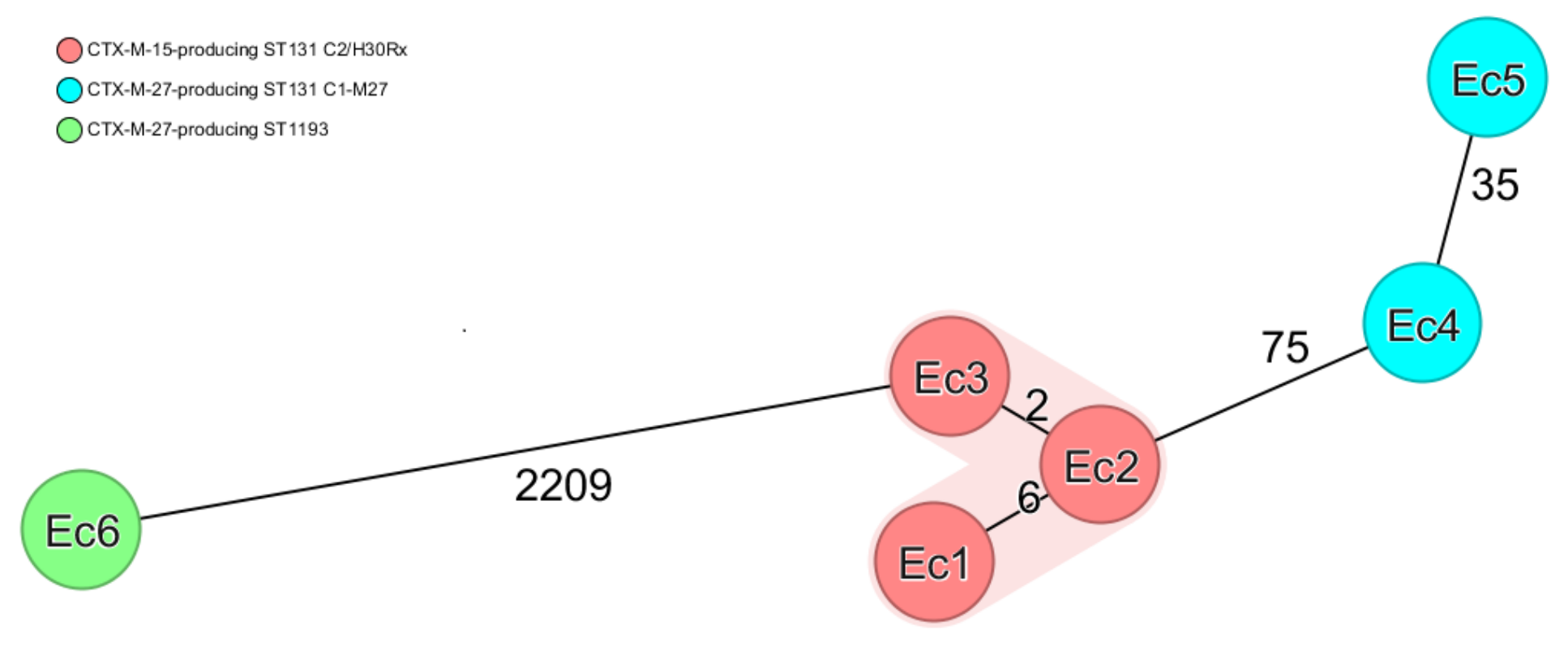

\section{Figure 2}

Core genome MLST based Minimum Spanning Tree of the ESBL-producing Escherichia coli isolates Figure 2 legend: Minimum spanning tree of Escherichia coli isolates based on the Ridom ${ }^{\text {TM }}$ SeqSphere+ core genome multilocus sequencing typing (cgMLST) including 2517 alleles with a cluster threshold $\leq 10$. Colors correspond with a different genotype characteristic and sequence type of the group of strains. Numbers of allele differences are indicated between the two nodes. 